\title{
On the Suitability of Suffix Arrays for Lempel-Ziv Data Compression
}

\author{
Artur J. Ferreira ${ }^{1,3}$ Arlindo L. Oliveira ${ }^{2,4}$ Mário A. T. Figueiredo ${ }^{3,4}$ \\ 1 Instituto Superior de Engenharia de Lisboa, Lisboa, PORTUGAL \\ 2 Instituto de Engenharia de Sistemas e Computadores: Investigação e \\ Desenvolvimento, Lisboa, PORTUGAL \\ 3 Instituto de Telecomunicações, Lisboa, PORTUGAL \\ ${ }^{4}$ Instituto Superior Técnico, Lisboa, PORTUGAL \\ arturj@cc.isel.ipl.pt aml@inesc-id.pt mario.figueiredo@lx.it.pt
}

\begin{abstract}
Lossless compression algorithms of the Lempel-Ziv (LZ) family are widely used nowadays. Regarding time and memory requirements, LZ encoding is much more demanding than decoding. In order to speed up the encoding process, efficient data structures, like suffix trees, have been used. In this paper, we explore the use of suffix arrays to hold the dictionary of the LZ encoder, and propose an algorithm to search over it. We show that the resulting encoder attains roughly the same compression ratios as those based on suffix trees. However, the amount of memory required by the suffix array is fixed, and much lower than the variable amount of memory used by encoders based on suffix trees (which depends on the text to encode). We conclude that suffix arrays, when compared to suffix trees in terms of the trade-off among time, memory, and compression ratio, may be preferable in scenarios (e.g., embedded systems) where memory is at a premium and high speed is not critical.
\end{abstract}

\section{Introduction}

Lossless compression algorithms of the Lempel-Ziv (LZ) family $[10,12,16]$ are widely used in a variety of applications. These coding techniques exhibit a high asymmetry in terms of time and memory requirements of the encoding and decoding processes, with the former being much more demanding due to the need to build, store, and search over a dictionary. Considerable research efforts have been devoted to speeding up LZ encoding procedures. In particular, efficient data structures have been suggested for this purpose; in this context, suffix trees (ST) $[3,8,13,14]$ have been proposed in $[5,6]$.

Recently, attention has been drawn to suffix arrays (SA), due to their simplicity and space efficiency. This class of data structures has been used in such diverse areas as search, indexing, plagiarism detection, information retrieval, biological sequence analysis, and linguistic analysis [9]. In data compression, SA have been used to encode data with anti-dictionaries [2] and optimized for large alphabets [11]. Linear-time SA construction algorithms have been proposed [4, 15]. The space requirement problem of the $\mathrm{ST}$ has been addressed by replacing an ST-based algorithm with another based on an enhanced $S A$ [1]. 
In this work, we show how an SA $[3,7]$ can replace an ST to hold the dictionary in the Lempel-Ziv 77 (LZ77) and Lempel-Ziv-Storer-Szymanski (LZSS) encoding algorithms. We also compare the use of ST and SA, regarding time and memory requirements of the data structures of the encoder.

The rest of the paper is organized as follows. Section 2 describes the LZ77 [16] algorithm and its variant LZSS [12]. Sections 3 and 4 present the main features of ST and SA, showing how to apply them to LZ77 compression. Some implementation details are discussed in Section 5. Experimental results are reported in Section 6. Some concluding remarks and future work details are discussed in Section 7.

\section{$2 \quad$ LZ77 and LZSS Compression}

The well-known LZ77 and LZSS encoding algorithms use a sliding window over the sequence of symbols, which has two sub-windows: the dictionary (holding the symbols already encoded) and the look-ahead-buffer (LAB), containing the symbols still to be encoded $[10,16]$. As a string of symbols in the LAB is encoded, the window slides to include it in the dictionary (this string slides in); consequently, symbols at the far end of the dictionary slide out.

At each step of the LZ77/LZSS encoding algorithm, the longest prefix of the LAB which can be found anywhere in the dictionary is determined and its position stored. In the example of Fig. 1, the string of the first four LAB symbols ("brow") is found in position 17 of the dictionary. For these two algorithms, encoding of a string consists in describing it by a token. The LZ77 token is a triplet of fields (pos, len, symbol), with the following meanings:

1. pos - location of the longest prefix of the LAB found in the current dictionary; this field uses $\log _{2}(\mid$ dictionary $\mid)$ bits, where |dictionary| is the length of the dictionary;

2. len - length of the matched string; this requires $\log _{2}(|\mathrm{LAB}|)$ bits;

3. symbol - the first symbol in the LAB, that does not belong to the matched string (i.e., that breaks the match); for ASCII symbols, this uses 8 bits.

In the absence of a match, the LZ77 token is $(0,0$, symbol $)$. For LZSS, the token has the format (bit,code), with code depending on value bit as follows:

$$
\left\{\begin{array}{l}
\text { bit }=0 \Rightarrow \text { code }=(\text { symbol }), \\
\text { bit }=1 \Rightarrow \text { code }=(\text { pos }, \text { len })
\end{array}\right.
$$

The idea is that, when a match exists, there is no need to explicitly encode the next symbol. If there is no match, LZSS produces $(0($ symbol $))$. Besides this modification, Storer and Szymanski [12] also proposed keeping the LAB in a circular queue and the dictionary in a binary search tree, to optimize the search. LZSS is widely used in practice (e.g., in GZIP and PKZIP), followed by entropy encoding, since it typically achieves higher compression ratios than LZ77.

Fig. 1 illustrates LZ77 and LZSS encoding. In LZ77, the string "brows" is encoded by $(17,4, \mathrm{~s})$; the window then slides 5 positions forward, thus the string 
"after" slides out, while the string "brows" slides in. In LZSS, "brow" is encoded as $(1(17,4))$ and "brow" slides in. Each LZ77 token uses $\log _{2}(\mid$ dictionary $\mid)+$ $\log _{2}(|\mathrm{LAB}|)+8$ bits; usually, $\mid$ dictionary $|\gg| \mathrm{LAB} \mid$. In LZSS, the token uses either 9 bits, when it has the form $(0,($ symbol $))$, or $1+\log _{2}(\mid$ dictionary $\mid)+$ $\log _{2}(|\mathrm{LAB}|)$ bits, when it has the form (1,(position,length) $)$.

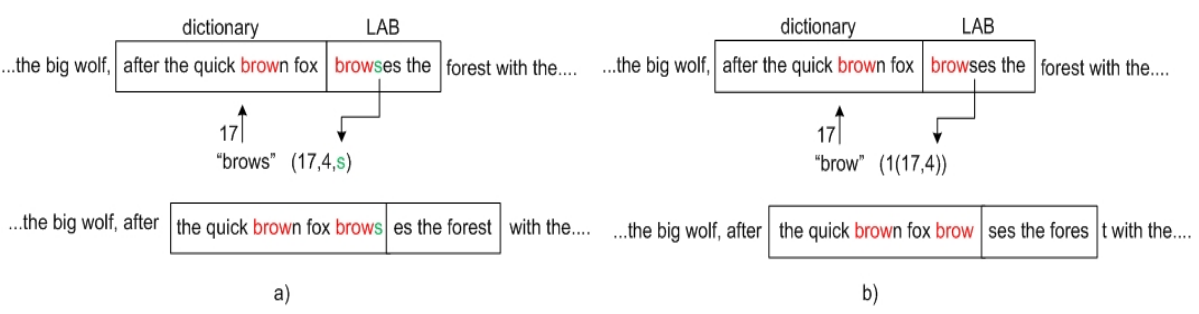

Fig. 1. a) LZ77 encoding of string "brows", with token $\left(17,4\right.$, 's' $\left.^{\prime}\right)$ b) LZSS encoding of string "brow", with token $(1(17,4))$.

The key component of the LZ77/LZSS encoding algorithms is the search for the longest match between LAB prefixes and the dictionary. Recently, ST have been used as efficient data structures to efficiently support this search [6].

\subsection{Decoding}

Assuming the decoder and encoder are initialized with equal dictionaries, the decoding of each LZ77 token (pos, len,symbol) proceeds as follows: (1) len symbols are copied from the dictionary to the output, starting at position pos of the dictionary; (2) the symbol symbol is appended to the output; (3) the string just produced at the output is slid into the dictionary.

For LZSS, we have: if the bit field is 1, len symbols, starting at position pos of the dictionary, are copied to the output; if it is 0 , symbol is copied to the output; finally, the string just produced at the output is slid into the dictionary.

Both LZ77/LZSS decoding are low complexity procedures. This work focuses only on encoder data structures and algorithms, with no effect in the decoder.

\section{Suffix Trees for LZ77 Compression}

A suffix tree (ST) is a data structure, built from a string, that contains the entire set of suffixes of that string $[3,8,13,14]$. Given a string $D$ of length $m$, an ST consists of a direct tree with $m$ leaves, numbered from 1 to $m$. Each internal node, except from the root node, has two or more descendants, and each branch corresponds to a non-empty sub-string of $D$. The branches stemming from the same node start with different symbols. For each leaf node, $i$, the concatenation of the strings over the branches, starting from the root to the leaf node $i$, yields the suffix of $D$ that starts at position $i$, that is, $D[i \ldots m]$. Fig. 2 shows the ST for 
string $D=x a b x a \$$, with suffixes xabxa\$, abxa\$, bxa\$, xa\$, a\$ and \$. Each leaf node contains the corresponding suffix number. In order to be possible to build

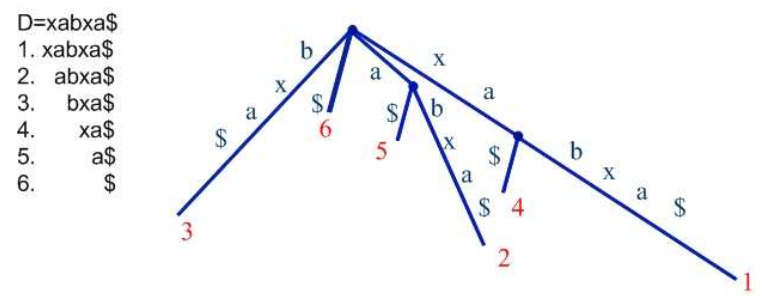

Fig. 2. Suffix Tree for string $D=x a b x a \$$. Each leaf node contains the corresponding suffix number. Each suffix is obtained by walking down the tree, from the root node.

an ST from a given string, it is necessary that no suffix of smaller length prefixes another suffix of greater length. This condition is assured by the insertion of a terminator symbol $(\$)$ at the end of the string. The terminator is a special symbol that does not occur previously on the string [3].

\subsection{Encoding Using Suffix Trees}

An ST can be applied to obtain the LZ77/LZSS encoding of a file, as we show in Algorithm 1 [3].

\begin{tabular}{l}
\hline Algorithm 1 - ST-Based LZ77 Encoding \\
Inputs: In, input stream to encode; $m$ and $n$, length of dictionary and LAB. \\
Output: Out, output stream with LZ77 description of $I n$. \\
\hline
\end{tabular}

1. Read dictionary $D$ and look-ahead-buffer $L A B$ from $I n$.

2. While there are symbols of In to encode:

a) Build, in $\mathcal{O}(m)$ time, an ST for string $D$.

b) Number each internal node $v$ with $c_{v}$, the smallest number of all the suffixes in $v$ 's subtree; this way, $c_{v}$ is the left-most position in $D$ of any copy of the sub-string on the path from the root to node $v$.

c) To obtain the description (pos, len) for the sub-string $L A B[i \ldots n]$, with $0 \leq i<n$ :

c1) If no suffix starts with $L A B[i]$, output $(0,0, L A B[i])$ to Out and do $i \leftarrow i+1$, and goto $2 \mathrm{c} 6)$.

c2) Follow the only path from the root that matches the prefix $L A B[i \ldots n]$.

c3) The traversal stops at point $p$ (not necessarily a node), when a symbol breaks the match; let $\operatorname{depth}(p)$ be the length of the string from the root to $p$ and $v$ the first node at or below $p$.

c4) Do pos $\leftarrow c_{v}$ and len $\leftarrow \operatorname{depth}(p)$.

c5) Output token (pos, len, $L A B[j]$ ) to $O u t$, with $j=i+$ len, do $i \leftarrow j+1$.

c6) if $i=n$ goto $2 \mathrm{~d}$ ); else goto $2 \mathrm{c}$ ). 
d) Slide in the full encoded LAB into $D$;

e) Read next LAB from In; goto 2).

The search in step 2c) of Algorithm 1 obtains the longest prefix of $L A B[i \ldots n]$ that also occurs in $D$. Fig. 3 shows the use of this algorithm for dictionary $D=a b b a c b b a \$$; every leaf node has the number of the corresponding suffix, while each internal node holds the corresponding $c_{v}$ value. In a similar manner,

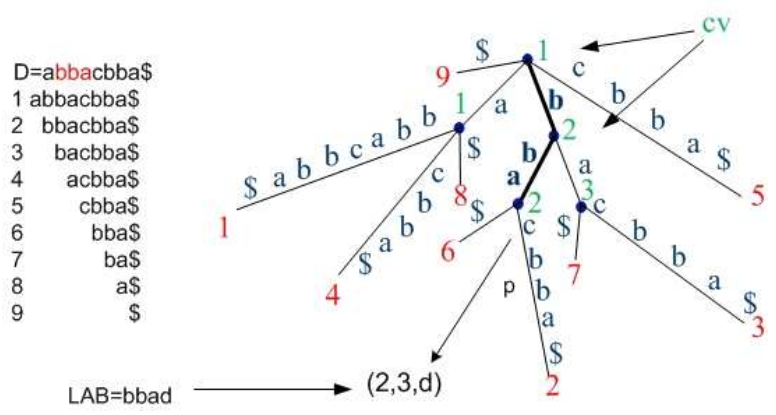

Fig. 3. Use of a Suffix Tree for LZ77 encoding, with dictionary $D=a b b a c b b a \$$. Each leaf node contains the corresponding suffix number, while the internal nodes keep the smallest suffix number on their subtree $\left(c_{v}\right)$. Point $p$ shows the end of the path that we follow, to encode the string $L A B=b b a d$.

the algorithm can be applied for LZSS compression, by modifying steps 2c1) and 2c5) in order to define the token, according to (1). Regarding Fig. 3, suppose that we want to encode the string $L A B=b b a d$; we traverse the tree from the root to point $p$ (with depth 3 ) and the closest node at or below $p$ has $c_{v}=2$, so the token has position $=2$ and length $=3$; this way, the token for $L A B$ on $D$ is $(2,3$, 'd') .

\section{Suffix Arrays for LZ77 Compression}

A suffix array (SA) is the lexicographically sorted array of the suffixes of a string $[3,7]$. For a string $D$ of length $m$ (with $m$ suffixes), an SA $P$ is a list of integers from 1 to $m$, according to the lexicographic order of the suffixes of $D$. For instance, if we consider $D=$ mississippi (with $m=11$ ), we get the suffixes in Fig. 4 part a); after sorting, we get the suffixes in part b). Thus, the SA for $D$ is $P=\{11,8,5,2,1,10,9,7,4,6,3\}$. Each of these integers is the suffix number, therefore corresponding to its position in $D$. Finding a sub-string of $D$ can be done by searching vector $P$; for instance, the set of sub-strings of $D$ that start with symbol 's', can be found at positions $7,4,6$, and 3 . As a result of this, an SA can be used to obtain every occurrence of a sub-string within a given string. 


$\begin{array}{ll} & \downarrow \\ \text { 1. mississippi } & \text { 11. i } \\ \text { 2. ississippi } & \text { 8. ippi } \\ \text { 3. ssissippi } & \text { 5. issippi } \\ \text { 4. sissippi } & \text { 2. ississippi } \\ \text { 5. issippi } & \text { 1. mississippi } \\ \text { 6. ssippi } & \text { 10. pi } \\ \text { 7. sippi } & \text { 9. ppi } \\ \text { 8. ippi } & \text { 7. sippi } \\ \text { 9. ppi } & \text { 4. sissippi } \\ \text { 10. pi } & \text { 6. ssippi } \\ \text { 11. i } & \text { 3. ssissippi }\end{array}$

a)

b)

Fig. 4. String $D=$ mississippi: a) Set of suffixes b) Sorted suffixes and SA $P$.

For LZ77/LZSS encoding, we can find the set of sub-strings of $D$, starting with a given symbol (the first in the LAB).

SA are an alternative to ST, as an SA implicitly holds the same information as an ST. Typically, it requires $3 \sim 5$ times less memory and can be used to solve the sub-string problem almost as efficiently as an ST [3]; moreover, its use is more appropriate when the alphabet is large. An SA can be built using a sorting algorithm, such as "quicksort"; it is possible to convert an ST into an SA in linear time [3]. An SA does not has the limitation of an ST: no suffix of smaller length prefixes another suffix of greater length.

The lexicographic order of the suffixes implies that suffixes that start with the same symbol are consecutive on SA $P$. This means that a binary search on $P$ can be used to find all these suffixes. This search takes $\mathcal{O}(n \log (m))$ time, with $n$ being the length of the sub-string to find, while $m$ is the length of the dictionary. To avoid some redundant comparisons on this binary search, the use of longest common prefix (LCP) of the suffixes, lowers the search time to $\mathcal{O}(n+\log (m))$ [3]. For a SA with $m$-length, the computation of its LCP takes $\mathcal{O}(m)$ time.

\subsection{Encoding Using Suffix Arrays}

Algorithm 2 shows how we can perform LZ77/LZSS encoding with an SA.

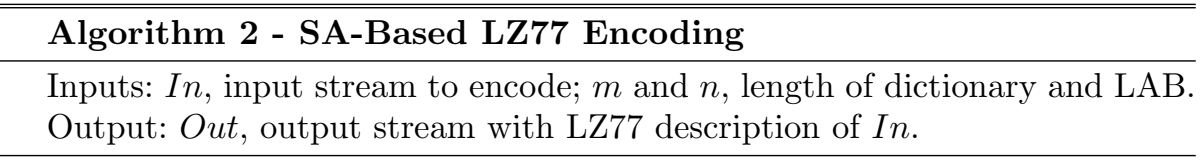

1. Read dictionary $D$ and look-ahead-buffer $L A B$ from $I n$.

2. While there are symbols of $I n$ to encode:

a) Build SA for string $D$ and name it $P$.

b) To obtain the description (pos,len) for every sub-string $L A B[i \ldots n], 0 \leq$ $i<n$, proceed as follows:

b1) If no suffix starts with $L A B[i]$, output $(0,0, L A B[i])$ to Out, set $i \leftarrow i+1$ and goto $2 \mathrm{~b} 5)$ 
b2) Do a binary search on vector $P$ until we find: the first position left, in which the first symbol of the corresponding suffix matches $L A B[i]$, that is, $D[P[$ left $]]=L A B[i]$; the last position right, in which the first symbol of the corresponding suffix matches $L A B[i]$, that is, $D[P[$ right $]]=L A B[i]$.

b3) From the set of suffixes between $P[l e f t]$ and $P[$ right $]$, choose the $k^{t h}$ suffix, left $\leq k \leq r i g h t$, with a given criteria (see below) giving a p-length match.

b4) Do pos $\leftarrow k$, len $\leftarrow p$ and $i \leftarrow i+l e n$ and output token (pos, len, $L A B[i])$ into Out.

b5) If $i=n$ goto $2 \mathrm{c}$ ); else goto $2 \mathrm{~b}$ ).

c) Slide in the full encoded LAB into $D$;

d) Read next LAB from In; goto 2).

In step 2.b2), it is possible to choose among several suffixes, according to a greedy/non-greedy parsing criterion. If we seek a fast search, we can choose one of the immediate suffixes, given by left or right. If we want better compression ratio, at the expense of a not so fast search, we should choose the suffix with the longest match with sub-string $L A B[i \ldots n]$. LZSS encoding can be done in a similar way, by changing the token format according to (1).

Fig. 5 illustrates LZ77 encoding with SA using dictionary $D=$ mississippi. We present two encoding situations: part a), in which we consider $L A B=i$ ssia encoded by $(5,4, a)$ or $(2,4, a)$, depending on how we perform the binary search and the choice of the match; part b), with $L A B=$ psi being encoded by $(10,1, s)$ or $(9,1, s)$ followed by $(0,0, i)$.
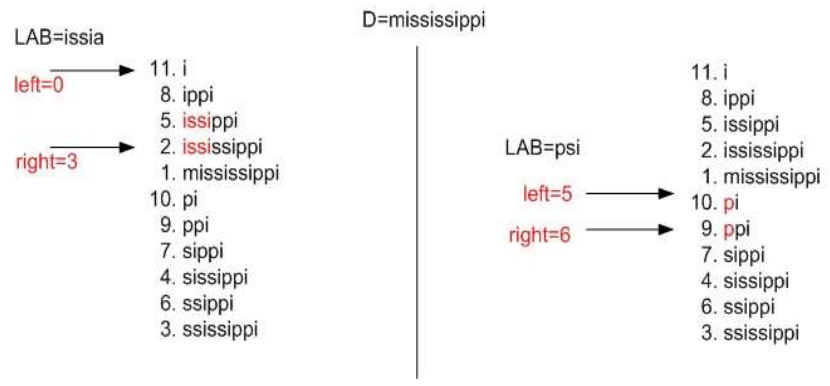

Fig. 5. LZ77/LZSS encoding with SA, with dictionary $D=$ mississippi, showing left and right indexes. In part a) we encode $L A B=i s s i a$, while in part b) we have $L A B=$ psi. 


\section{Implementation Details}

We have considered a memory-efficient ST representation ${ }^{5}$, that when compared to others, has the smallest memory requirement for the ST data structures. This implementation (written in $\mathrm{C}++$ ) builds a tree from a string using Ukkonen's algorithm [3,13] and has the following main features: it holds a single version of the string; the tree is composed of branches and nodes; uses an hash table to store the branches and an array for the nodes; the Hash is computed as a function of the number of the node and the first symbol of the string on that branch, as depicted in Fig. 6. Two main changes in this source code were made:

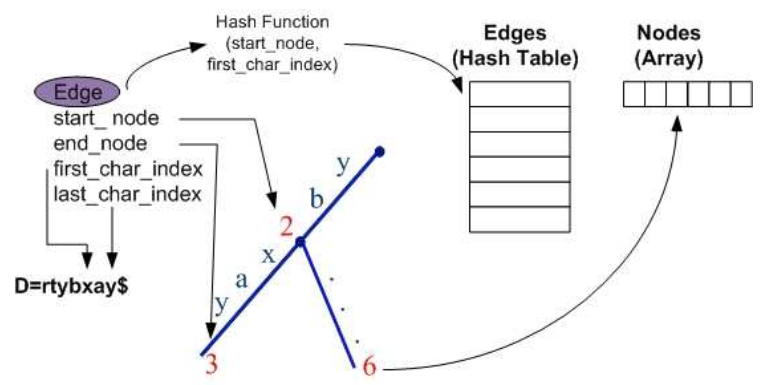

Fig. 6. Illustration of ST data structures. The branch between nodes 2 and 3, with string "xay" that starts and ends at positions 4 and 6 , respectively. The node only contains its suffix link; we have added the number of its parent and the counter $c_{v}$ to each node as described in Algorithm 1, subsection 3.1.

the Node was equipped with the number of its parent and with the counter $c_{v}$; using the number of its parent, the code for the propagation of the $c_{v}$ values from a node was written. After these changes we wrote Algorithm 1, as described in subsection 3.1.

For SA computation, we have used the package, written in 'C' programming language, available at http://www.cs.dartmouth.edu/ doug/sarray/, which includes functions to compute SA and LCP.

int sarray (int $* a$, int $\mathrm{m}$ );

int $* 1 \mathrm{lp}($ const int $* \mathrm{a}$, const char $* \mathrm{~s}$, int $\mathrm{m})$;

The SA computation takes $\mathcal{O}(m \log (m))$ time, while for LCP we have $\mathcal{O}(m)$ time. Using these functions, we wrote Algorithm 2, described in subsection 4.1. For both encoders (with ST and SA), we copy the contents of the LAB to the end of the dictionary, only when the entire contents of the LAB is encoded. Another important issue is the fact that for ST (but not for the SA), we add a terminator symbol to end of the dictionary, to assure that we have a valid ST (no suffix of smaller length prefixes another suffix of greater length). This justifies the small differences in the compression ratios attained by the ST and SA encoders.

\footnotetext{
${ }^{5}$ http://marknelson.us/1996/08/01/suffix-trees/
} 


\section{Experimental Results}

This section presents the experimental results of LZSS encoders, with dictionaries and LAB of different lengths. The evaluation was carried out using standard test files from the well-known Calgary Corpus ${ }^{6}$ and Canterbury Corpus ${ }^{7}$. We have considered a set of 18 files, with a total size of 2680580 bytes: bib (111261 bytes); book1 (768771 bytes); book2 (610856 bytes); news (377109 bytes); paper1 (53161 bytes); paper2 (82199 bytes); paper3 (46526 bytes); paper4 (13286 bytes); paper5 (11954 bytes); paper6 (38105 bytes); progc (39611 bytes); progl (71646 bytes); progp (49379 bytes); trans (93695 bytes); alice29 (152089 bytes); asyoulik (125179 bytes); cp (24603 bytes); fields (11150 bytes).

The compression tests were executed on a machine with 2GB RAM and an Intel processor Core2 Duo CPU T7300 @ 2GHz. We measured the following parameters: encoding time (in seconds), memory occupied by the encoder data structures (in bytes) and compression ratio in bits per byte

$$
b p b=8 \frac{\text { Encoded Size }}{\text { Original Size }},
$$

for the LZSS encoder. The memory indicator refers to the average amount of memory needed for every ST and SA, built in the encoding process, given by

$$
\begin{aligned}
& M_{S T}=\mid \text { Edges }|+| \text { Nodes }|+| \text { dictionary }|+| \mathrm{LAB} \mid, \\
& M_{S A}=\mid \text { Suffix Array }|+| \text { dictionary }|+| \mathrm{LAB} \mid,
\end{aligned}
$$

for the ST encoder and SA encoder, respectively (the operator |.| gives the length in bytes). Each ST edge is made up of 4 integers (see Fig. 6) and each node has 3 integers (suffix link, $c_{v}$ counter and parent identification, as described in Section 5); each integer occupies 4 bytes. On the SA encoder tests, we have considered the choice of $k$ as the mid-point between left and right, as stated in Algorithm 2 in subsection 4.1.

\subsection{Encoding Time, Memory and Compression Ratio}

We consider a small dictionary with length 128 and $|\mathrm{LAB}|=16$ or $|\mathrm{LAB}|=32$, as shown in Table 1 with the results for the 18 chosen files. We see that SA are slightly faster than ST, but achieve a lower compression ratio. The amount of memory for the SA encoder is fixed at $660\left(=129^{*} 4+128+16\right)$ and 676 $\left(=129^{*} 4+128+32\right)$. The amount of memory for the ST encoder is larger and variable, because the number of edges and nodes depends on the suffixes of the string in the dictionary. With small dictionaries, we have a low encoding time (fast compression) with a reasonable compression ratio. We conclude that the increase of the LAB gives rise to a lower encoding time, for both ST and SA encoders. For $|\mathrm{LAB}|=32$, the SA encoder is faster than the ST encoder, but this last one attains a better compression ratio.

\footnotetext{
${ }^{6}$ http://links. uwaterloo.ca/calgary.corpus.html

7 http://corpus. canterbury.ac.nz/
} 
Table 1. LZSS encoding time (in seconds), memory and compression results for the set of 18 files, with $\mid$ dictionary $|=128,| \mathrm{LAB} \mid=16$ and $\mid$ dictionary $|=128,| \mathrm{LAB} \mid=32 . M_{S T}$ and $M_{S A}$ are as defined in (3), and bpb is as defined in (2).

\begin{tabular}{|l|c|c|c|c|c|c|c|c|c|c|c|c|}
\multicolumn{1}{c|}{} & \multicolumn{3}{|c|}{$\mid$ dictionary $|=\mathbf{1 2 8}| \mathbf{L A B} \mid=\mathbf{1 6}$} & \multicolumn{3}{c|}{$\mid$ dictionary $|=\mathbf{1 2 8}| \mathbf{L A B} \mid=\mathbf{3 2}$} \\
\cline { 2 - 15 } \multicolumn{1}{c|}{} & \multicolumn{3}{|c|}{ ST } & \multicolumn{3}{|c|}{ SA } & \multicolumn{3}{c|}{ SA } \\
\hline File & Time & $M_{S T}$ & bpb & Time & $M_{S A}$ & bpb & Time & $M_{S T}$ & bpb & Time & $M_{S A}$ & bpb \\
\hline bib & 2.8 & 4888 & 5.01 & 2.9 & 660 & 6.27 & 1.3 & 4904 & 5.19 & 1.5 & 676 & 6.23 \\
\hline book1 & 23.3 & 4888 & 4.73 & 20.4 & 660 & 5.76 & 11.5 & 4932 & 4.94 & 10.0 & 676 & 5.80 \\
\hline book2 & 18.7 & 5980 & 4.56 & 16.2 & 660 & 5.50 & 9.0 & 5996 & 4.74 & 7.9 & 676 & 5.53 \\
\hline news & 10.6 & 6372 & 4.94 & 10.0 & 660 & 5.98 & 5.1 & 6388 & 5.11 & 4.9 & 676 & 6.04 \\
\hline paper1 & 1.6 & 4748 & 4.62 & 1.4 & 660 & 5.56 & 0.8 & 4876 & 4.81 & 0.7 & 676 & 5.62 \\
\hline paper2 & 2.6 & 5000 & 4.60 & 2.2 & 660 & 4.76 & 1.2 & 5044 & 4.78 & 1.1 & 676 & 5.58 \\
\hline paper3 & 1.4 & 4916 & 4.70 & 1.2 & 660 & 5.69 & 0.7 & 5072 & 4.88 & 0.6 & 676 & 5.72 \\
\hline paper4 & 0.4 & 4944 & 4.64 & 0.4 & 660 & 5.60 & 0.2 & 5044 & 4.79 & 0.2 & 676 & 5.60 \\
\hline paper5 & 0.3 & 5252 & 4.60 & 0.3 & 660 & 5.52 & 0.2 & 5408 & 4.79 & 0.2 & 676 & 5.56 \\
\hline paper6 & 1.1 & 5336 & 4.51 & 1.1 & 660 & 5.38 & 0.6 & 5352 & 4.70 & 0.5 & 676 & 5.44 \\
\hline progc & 1.1 & 5924 & 4.37 & 1.1 & 660 & 5.09 & 0.5 & 6220 & 4.48 & 0.5 & 676 & 5.07 \\
\hline progl & 2.2 & 5336 & 4.08 & 2.0 & 660 & 4.39 & 1.1 & 5296 & 4.07 & 1.0 & 676 & 4.36 \\
\hline progp & 1.4 & 5364 & 4.00 & 1.3 & 660 & 4.38 & 0.7 & 5380 & 3.99 & 0.7 & 676 & 4.40 \\
\hline trans & 2.7 & 7212 & 4.45 & 2.5 & 660 & 5.34 & 1.3 & 7228 & 4.57 & 1.3 & 676 & 5.34 \\
\hline alice29 & 4.5 & 5392 & 4.60 & 4.1 & 660 & 5.45 & 2.3 & 5408 & 4.75 & 2.0 & 676 & 5.48 \\
\hline asyoulik & 3.7 & 5028 & 4.64 & 3.3 & 660 & 5.57 & 1.8 & 5044 & 4.82 & 1.6 & 676 & 5.60 \\
\hline cp & 0.7 & 5308 & 4.64 & 0.7 & 660 & 5.86 & 0.3 & 5184 & 4.70 & 0.3 & 676 & 5.69 \\
\hline fields & 0.3 & 6036 & 3.90 & 0.3 & 660 & 4.30 & 0.2 & 6080 & 3.96 & 0.2 & 676 & 4.24 \\
\hline \hline Average & $\mathbf{4 . 4}$ & $\mathbf{5 4 4 0}$ & $\mathbf{4 . 4 7}$ & $\mathbf{4 . 0}$ & $\mathbf{6 6 0}$ & $\mathbf{5 . 3 5}$ & $\mathbf{2 . 2}$ & $\mathbf{5 4 9 2}$ & $\mathbf{4 . 6 7}$ & $\mathbf{1 . 9}$ & $\mathbf{6 7 6}$ & $\mathbf{5 . 4 0}$ \\
\hline Total & $\mathbf{7 9 . 4}$ & $\mathbf{9 7 9 2 4}$ & & $\mathbf{7 1 . 3}$ & $\mathbf{1 1 8 8 0}$ & & $\mathbf{3 8 . 8}$ & $\mathbf{9 8 8 5 6}$ & & $\mathbf{3 5 . 0}$ & $\mathbf{1 2 1 6 8}$ & \\
\hline
\end{tabular}

We repeat the tests of Table 1, but with larger dictionary and LAB lengths. First, we consider $\mid$ dictionary $\mid=512$ and $|\mathrm{LAB}|=128$, giving $2692=513^{*} 4+512$ +128 bytes for the encoder data structures; we also used |dictionary|=1024 and $|\mathrm{LAB}|=256$. Comparing the results in Table 2 with those in Table 1, we see that the encoding time decreases when the dictionary and LAB increase their length. The dictionary and LAB lengths in Table 2 achieve better compression ratio.

Table 3 presents the average values for the set of 18 files, using different combinations of dictionary and LAB lengths. As the length of the dictionary increases, the ST-encoder takes more time and uses much more memory than the SA-encoder. The encoding time of the SA-encoder does not grow as fast as for the ST-encoder, along with the length of the dictionary. For both encoders, we see that the worst scenarios are those in which we have a large dictionary and a small $\mathrm{LAB}$, like in the $\mid$ dictionary $\mid=1024$ and $|\mathrm{LAB}|=8$ test. On the other hand, for $\mid$ dictionary $\mid=1024$ and $|\mathrm{LAB}|=256$ we get a very reasonable encoding time, being faster than the ST-encoder. It is important to notice that our ST implementation is not fully optimized in terms of speed, but it is memory-efficient. There are other implementations which are more time-efficient, at the expense of memory 
Table 2. LZSS encoding time (in seconds), memory and compression results for the set of 18 files, with $\mid$ dictionary $|=512,| \mathrm{LAB} \mid=128$ and $\mid$ dictionary $|=1024,| \mathrm{LAB} \mid=256$.

\begin{tabular}{|l|c|c|c|c|c|c|c|c|c|c|c|c|}
\cline { 2 - 15 } \multicolumn{1}{c|}{} & \multicolumn{3}{|c|}{$\mid$ dictionary $|=\mathbf{5 1 2 ,}| \mathbf{L A B} \mid=\mathbf{1 2 8}$} & \multicolumn{3}{c|}{$\mid$ dictionary $|=\mathbf{1 0 2 4}| \mathbf{L A B} \mid=\mathbf{2 5 6}$} \\
\cline { 2 - 15 } & \multicolumn{3}{|c|}{ ST } & \multicolumn{3}{|c|}{ SA } & \multicolumn{3}{|c|}{ ST } & \multicolumn{3}{c|}{ SA } \\
\hline File & Time & $M_{S T}$ & bpb & Time & $M_{S A}$ & bpb & Time & $M_{S T}$ & bpb & Time & $M_{S A}$ & $\mathrm{bpb}$ \\
\hline bib & 1.42 & 22100 & 4.73 & 0.55 & 2692 & 4.55 & 1.26 & 44468 & 4.82 & 0.55 & 5380 & 4.39 \\
\hline book1 & 11.19 & 20364 & 5.02 & 4.06 & 2692 & 5.03 & 11.23 & 42284 & 5.04 & 4.97 & 5380 & 4.93 \\
\hline book2 & 8.70 & 21092 & 4.67 & 4.06 & 2692 & 5.03 & 9.09 & 43600 & 4.70 & 3.48 & 5380 & 4.47 \\
\hline news & 4.56 & 22660 & 5.30 & 1.89 & 2692 & 5.32 & 4.75 & 43908 & 5.22 & 2.09 & 5380 & 5.03 \\
\hline paper1 & 0.73 & 21204 & 4.75 & 0.25 & 2692 & 4.69 & 0.77 & 45868 & 4.79 & 0.30 & 5380 & 4.54 \\
\hline paper2 & 1.22 & 20504 & 4.73 & 0.42 & 2692 & 4.67 & 1.30 & 42368 & 4.77 & 0.48 & 5380 & 4.58 \\
\hline paper3 & 0.66 & 20336 & 4.94 & 0.25 & 2692 & 4.90 & 0.72 & 42480 & 5.00 & 0.27 & 5380 & 4.82 \\
\hline paper4 & 0.19 & 21176 & 4.90 & 0.06 & 2692 & 4.84 & 0.20 & 44188 & 5.34 & 0.08 & 5380 & 5.12 \\
\hline paper5 & 0.16 & 23080 & 5.14 & 0.06 & 2692 & 5.03 & 0.16 & 47240 & 5.53 & 0.08 & 5380 & 5.27 \\
\hline paper6 & 0.52 & 22716 & 4.71 & 0.19 & 2692 & 4.62 & 0.55 & 44524 & 4.81 & 0.22 & 5380 & 4.56 \\
\hline progc & 0.48 & 20476 & 4.70 & 0.19 & 2692 & 4.52 & 0.52 & 42088 & 4.91 & 0.20 & 5380 & 4.54 \\
\hline progl & 0.97 & 22884 & 4.01 & 0.34 & 2692 & 3.68 & 0.97 & 45616 & 4.05 & 0.36 & 5380 & 3.56 \\
\hline progp & 0.63 & 21232 & 4.03 & 0.23 & 2692 & 3.73 & 0.64 & 44272 & 4.01 & 0.25 & 5380 & 3.54 \\
\hline trans & 1.28 & 23220 & 4.75 & 0.45 & 2692 & 4.73 & 1.31 & 45476 & 4.79 & 0.39 & 5380 & 4.53 \\
\hline alice29 & 2.02 & 21344 & 4.72 & 0.81 & 2692 & 4.61 & 2.05 & 42788 & 4.77 & 0.92 & 5380 & 4.51 \\
\hline asyoulik & 1.55 & 22016 & 4.86 & 0.66 & 2692 & 4.82 & 1.64 & 43768 & 4.93 & 0.77 & 5380 & 4.77 \\
\hline cp & 0.30 & 21092 & 4.52 & 0.11 & 2692 & 4.29 & 0.31 & 43292 & 4.81 & 0.13 & 5380 & 4.26 \\
\hline fields & 0.16 & 22212 & 4.36 & 0.05 & 2692 & 3.92 & 0.16 & 45336 & 4.71 & 0.06 & 5380 & 4.07 \\
\hline Average & $\mathbf{2 . 0 4}$ & $\mathbf{2 1 6 5 0}$ & $\mathbf{4 . 7 1}$ & $\mathbf{0 . 7 6}$ & $\mathbf{2 6 9 2}$ & $\mathbf{4 . 5 9}$ & $\mathbf{2 . 0 9}$ & $\mathbf{4 4 0 8 6}$ & $\mathbf{4 . 8 3}$ & $\mathbf{0 . 8 7}$ & $\mathbf{5 3 8 0}$ & $\mathbf{4 . 5 3}$ \\
\hline Total & $\mathbf{3 6 . 7 2}$ & $\mathbf{3 8 9 7 0 8}$ & & $\mathbf{1 3 . 7 0}$ & $\mathbf{4 8 4 5 6}$ & & $\mathbf{3 7 . 6 3}$ & $\mathbf{7 9 3 5 6 4}$ & & $\mathbf{1 5 . 5 9}$ & $\mathbf{9 6 8 4 0}$ & \\
\hline
\end{tabular}

usage; for instance, Larsson's ST-encoder ${ }^{8}$, uses 3 integers and a symbol for each node, placed in an hash table; for $\mid$ dictionary $\mid=256$ and $\mid$ dictionary $\mid=1024$, this implementation occupies for the encoder data structures, 7440 and 29172 bytes, respectively .

\subsection{Encoding Time and Memory Analysis}

Fig. 7 shows how encoding time varies with the length of the dictionary and LAB, for the set of 18 files. We have considered the 16 length combinations given by $\mid$ dictionary $\mid \in\{256,512,1024,2048\}$ and $|\mathrm{LAB}| \in\{8,16,32,128\}$. The $\mathrm{ST}$ encoder denotes higher increase in the encoding time for larger dictionaries and smaller LAB; for the SA encoder, this time increase is smaller. In order to obtain a reasonable encoding time, the length of the LAB should not be too small, as compared to the length of the dictionary. Fig. 8 displays the amount of memory as a function of the length of the dictionary and the LAB. The amount of memory does not change significantly with the length of the LAB. It increases with the length of the dictionary; this increase is clearly higher for the $\mathrm{ST}$ encoder. The compression ratio as a function of the length of the dictionary

\footnotetext{
${ }^{8}$ http://www . larsson.dogma.net/research.html
} 
Table 3. LZSS average encoding time, average memory and compression results for different lengths of dictionary and LAB, over the set of 18 files, for each pair |dictionary|, $|\mathrm{LAB}|$. The best results, regarding encoding time and compression ratio are underlined.

\begin{tabular}{|l|l|c|c|c|c|c|c|}
\cline { 2 - 8 } \multicolumn{2}{c|}{} & \multicolumn{3}{c|}{ ST } & \multicolumn{3}{c|}{ SA } \\
\hline \hline dictionary & LAB & Time & $M_{S T}$ & bpb & Time & $M_{S A}$ & bpb \\
\hline \hline 128 & 8 & $\underline{\mathbf{3 . 6}}$ & 5424 & $\underline{\mathbf{4 . 6 0}}$ & 8.1 & 652 & 5.57 \\
\hline 128 & 16 & $\underline{\mathbf{1 . 7}}$ & 5440 & $\underline{\mathbf{4 . 5 3}}$ & 3.9 & 660 & 5.40 \\
\hline 256 & 8 & 15.3 & 11015 & $\underline{\mathbf{4 . 5 0}}$ & $\underline{\mathbf{8 . 6}}$ & 1292 & 4.88 \\
\hline 256 & 16 & 7.1 & 11011 & $\underline{\mathbf{4 . 3 2}}$ & $\underline{\mathbf{4 . 0}}$ & 1300 & 4.67 \\
\hline 512 & 8 & 34.4 & 21776 & 4.55 & $\underline{\mathbf{9 . 4}}$ & 2572 & $\mathbf{4 . 5 1}$ \\
\hline 512 & 16 & 15.8 & 21785 & 4.27 & $\underline{\mathbf{4 . 5}}$ & 2580 & $\underline{\mathbf{4 . 2 6}}$ \\
\hline 512 & 128 & 2.1 & 21650 & 4.71 & $\underline{\mathbf{0 . 8}}$ & 2692 & $\underline{\mathbf{4 . 5 9}}$ \\
\hline 1024 & 8 & 70.3 & 44136 & 4.64 & $\underline{\mathbf{1 1 .}}$ & 5132 & $\underline{\mathbf{4 . 3 2}}$ \\
\hline 1024 & 16 & 32.8 & 44134 & 4.27 & $\underline{\mathbf{5 . 5}}$ & 5140 & $\mathbf{4 . 0 3}$ \\
\hline 1024 & 32 & 16.6 & 44132 & 4.21 & $\underline{\mathbf{3 . 0}}$ & 5156 & $\underline{\mathbf{3 . 9 9}}$ \\
\hline 1024 & 256 & 2.1 & 44086 & 4.83 & $\underline{\mathbf{0 . 9}}$ & 5380 & $\underline{\mathbf{4 . 5 3}}$ \\
\hline 2048 & 32 & 32.7 & 88856 & 4.28 & $\underline{\mathbf{4 . 5}}$ & 10276 & $\underline{\mathbf{3 . 9 3}}$ \\
\hline 2048 & 1024 & $\underline{\mathbf{1 . 1}}$ & 90235 & $\underline{\mathbf{4 . 2 8}}$ & 1.7 & 11268 & 5.00 \\
\hline 4096 & 1024 & $\underline{\mathbf{2 . 2}}$ & 181984 & 5.58 & 4.8 & 21508 & $\underline{\mathbf{5 . 0 9}}$ \\
\hline
\end{tabular}

and the LAB is displayed in Fig. 9. The difference between compression ratio is almost meaningless, when compared to the differences in time and memory.
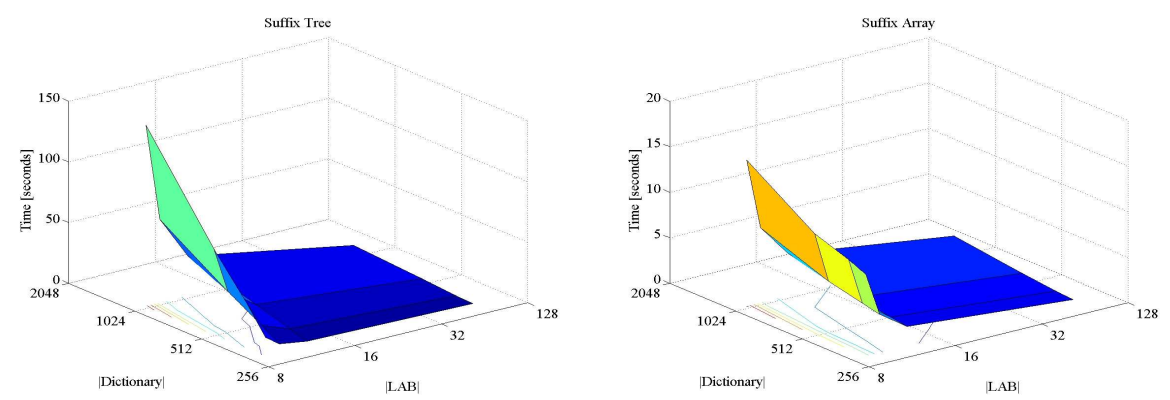

Fig. 7. Average encoding time (in seconds) as a function of the length of the dictionary and LAB, for ST and SA encoder, on the tests of Table 3.

\section{Conclusions and Future Work}

In this work, we have explored the use of suffix trees (ST) and suffix arrays (SA) for the Lempel-Ziv 77 family of data compression algorithms, namely LZ77 and LZSS. The use of ST and SA for the encoder was evaluated in different scenarios, using standard test files of different types and sizes, commonly used in embedded 

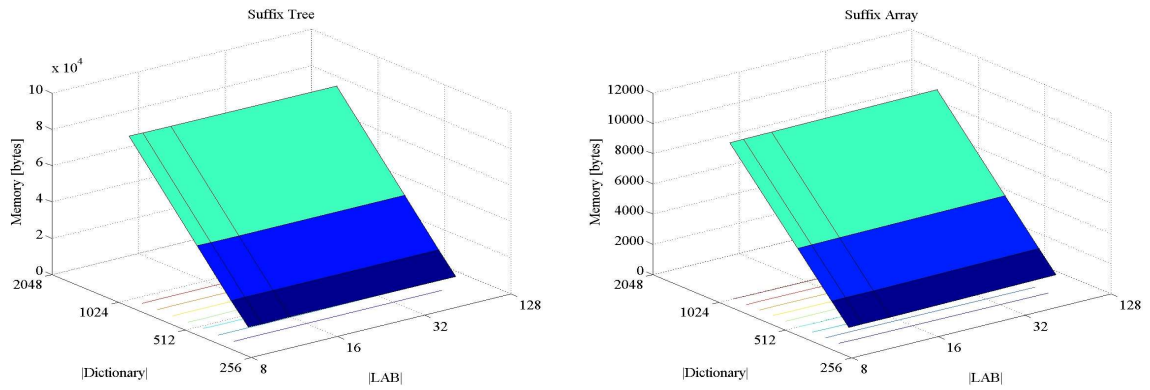

Fig. 8. Average memory (in $\mathrm{kB}$ ) as a function of the length of the dictionary and LAB, for ST and SA encoder, on the tests of Table 3.
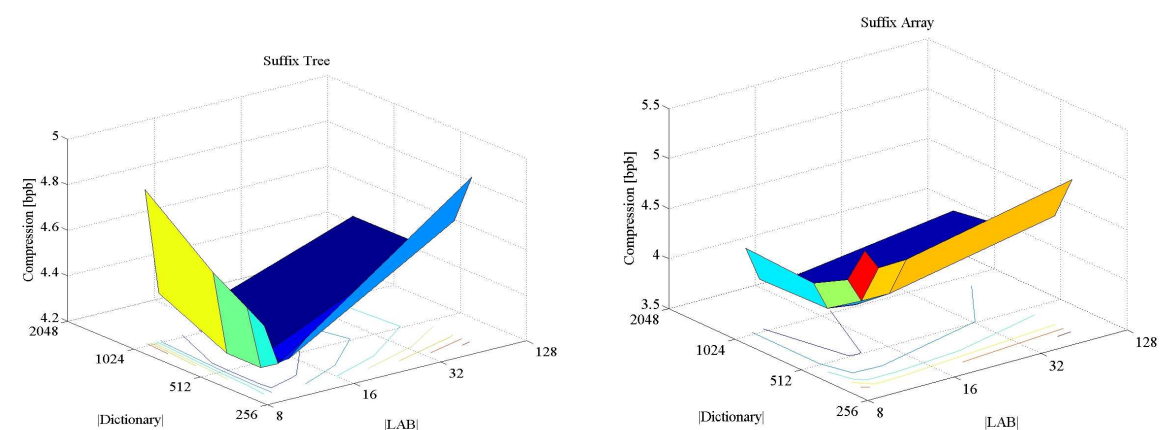

Fig. 9. Compression ratio (in bpb) as a function of the length of the dictionary and $\mathrm{LAB}$, for ST and SA encoder, on the tests of Table 3.

systems. Naturally, we focused on the encoder side, in order to see how we could perform an efficient search without spending too much memory. A comparison between a memory efficient implementation of ST and our SA encoders was carried out, using the following metrics: encoding time, memory requirement, and compression ratio. Our main conclusions are:

1. ST-based encoders require more memory than the SA counterparts;

2. the memory requirement of ST- and SA-based encoders is linear with the dictionary size; for the SA-based encoders, it does not depend on the contents of the file to be encoded;

3. for small dictionaries, there is no significant difference in terms of encoding time and compression ratio, between $\mathrm{ST}$ and $\mathrm{SA}$;

4. for larger dictionaries, ST-based encoders are slower that SA-based ones; however, in this case, the compression ratio with ST is slightly better than the one with SA.

These results support the claim that the use of SA is a very competitive choice when compared to ST, for Lempel-Ziv compression. We know exactly the memory requirement of the SA, which depends on the dictionary length and does 
not depend on the text to encode. In application scenarios where the length of the dictionary is medium or large and the available memory is scarce and speed is not so important, it is preferable to use SA instead of ST. This way, SA are suited for this purpose, regarding the trade-off between time, memory, and compression ratio, being preferable for mobile devices and embedded systems.

As ongoing and future work, we are developing two new faster SA-based algorithms. The first algorithm updates the indexes of the unique SA, built at the beginning of the encoding process, after each full look-ahead-buffer encoding. The idea is that after each look-ahead-buffer encoding, the dictionary is closely related to the previous one; this way, regarding the dictionary data structures we need only to update an array of integers. The second algorithm uses longest common prefix to get the length field of the token; this version will be more costly in terms of memory, but it should run faster than all the other SA-encoders.

\section{References}

1. Abouelhoda, M., Kurtz, S., and Ohlebusch, E. (2004). Replacing suffix trees with enhanced suffix arrays. Journal of Discrete Algorithms, 2(1):53-86.

2. Fiala, M. and Holub, J. (2008). DCA using suffix arrays. In Data Compression Conference DCC2008, page 516.

3. Gusfield, D. (1997). Algorithms on Strings, Trees and Sequences. Cambridge University Press.

4. Karkainen, J., Sanders, P., and S.Burkhardt (2006). Linear work suffix array construction. Journal of the ACM, 53(6):918-936.

5. Larsson, N. (1996). Extended application of suffix trees to data compression. In Data Compression Conference, page 190.

6. Larsson, N. (1999). Structures of String Matching and Data Compression. PhD thesis, Department of Computer Science, Lund University, Sweden.

7. Manber, U. and Myers, G. (1993). Suffix arrays: a new method for on-line string searches. SIAM Journal on Computing, 22(5):935-948.

8. McCreight, E. (1976). A space-economical suffix tree construction algorithm. Journal of the ACM, 23(2):262-272.

9. Sadakane, K. (2000). Compressed text databases with efficient query algorithms based on the compressed suffix array. In ISAAC'00, volume LNCS 1969, pages 410421.

10. Salomon, D. (2007). Data Compression - The complete reference. Springer-Verlag London Ltd, London, fourth edition.

11. Sestak, R., Lnsk, J., and Zemlicka, M. (2008). Suffix array for large alphabet. In Data Compression Conference DCC2008, page 543.

12. Storer, J. and Szymanski, T. (1982). Data compression via textual substitution. Journal of ACM, 29(4):928-951.

13. Ukkonen, E. (1995). On-line construction of suffix trees. Algorithmica, 14(3):249260.

14. Weiner, P. (1973). Linear pattern matching algorithm. In 14th Annual IEEE Symposium on Switching and Automata Theory, volume 27, pages 1-11.

15. Zhang, S. and Nong, G. (2008). Fast and space efficient linear suffix array construction. In Data Compression Conference DCC2008, page 553.

16. Ziv, J. and Lempel, A. (1977). A universal algorithm for sequential data compression. IEEE Transactions on Information Theory, IT-23(3):337-343. 